\title{
Hippocampal Representation in Place Learning
}

\author{
Howard Eichenbaum, ${ }^{1}$ Caroline Stewart, ${ }^{2}$ and R. G. M. Morris ${ }^{2}$ \\ 'Department of Biological Sciences, Wellesley College, Wellesley, Massachusetts 02181, and 2Department of \\ Pharmacology, University of Edinburgh Medical School, Edinburgh EH8 9JZ, Scotland
}

The generality of the place-learning impairment associated with hippocampal system damage was challenged using methods of training that permitted subjects to form an individual association between the place of escape and a particular navigational route in an open-field water maze. Both normal rats and rats with fornix lesions (FX rats) acquired this task rapidly, although $F X$ rats were slightly slower in achieving minimum escape latencies. In postcriterion testing, FX rats occasionally made near misses but, more often, their escape performance was indistinguishable from that of intact rats. Results from a variety of probe tests indicated that FX rats, like normal rats, had based their performance on a representation of multiple distal cues but their representation, unlike that of normal rats, was inflexible in that it could not be used to guide performance when the cues or starting position were altered.

These results parallel those from other studies of hippocampal function in animals and humans: The learning deficit consequent to hippocampal system damage (1) is not specific to a particular category of learning materials, but is dependent on the representational demands of the task; (2) is observed when task demands encourage a representation based on relations among multiple cues, but not when the task encourages adaptation to an individual (or compound) stimulus; (3) spares acquisition of fundamental procedures needed to perform the task; and (4) impairs the flexible use of learned information in tests other than repetition of the learning experience.

Considerable data support the hypothesis that the hippocampus is selectively or disproportionately involved in spatial learning and memory, at lcast in rats (O'Keefe and Nadel, 1978). However, in recent years, several investigators have demonstrated that, under some task conditions, hippocampal system damage results in severe memory impairment with nonspatial learning materials in humans (e.g., Graf et al., 1984), monkeys (e.g., Gaffan, 1974; Mishkin et al., 1983; Zola-Morgan and Squire, 1985), and rats (e.g., Olton and Feustle, 1981; Ross et al., 1984). One possible explanation of this pattern of results is that the hippocampal system is critical to a certain type of memory representation that is especially prominent in, but not unique

Received Feb. 16, 1990; revised June 25, 1990; accepted June 26, 1990.

We acknowledge the generous support of NSF grants BNS8721157 and BNS8810095, NIH grant NS18744, and a sabbatical grant from Wellesley College to H.E., and grants from the MRC and British Council to R.G.M.M. We also express our gratitude to Elma Forrest for performing the histological analyses in this experiment.

Correspondence should be addressed to Howard Eichenbaum at the above address.

Copyright (C) 1990 Society for Neuroscience $0270-6474 / 90 / 113531-12 \$ 03.00 / 0$ to, spatial learning. The aim of the present investigation is to clarify those aspects of spatial learning that are impaired and spared by hippocampal system damage and, by comparison to reports on nonspatial learning, reveal properties of hippocampal processing common across behavioral paradigms and across species.

Based on studies of nonspatial discrimination learning, Eichenbaum and colleagues $(1986,1988,1989)$ proposed that hippocampal processing is critical for memory representations of relations among multiple independent cues, but is not required for learning that can be based on adaptations to individual cues. They suggested that, in intact animals, a relational representation can be identified by its flexibility, that is, its ability to support the use of learned discriminative cues in novel situations. Conversely, in the absence of a relational representation, adaptations to individual discriminative stimuli can be revealed only through improved performance in repetitions of the original learning event. These characterizations of hippocampal involvement in discrimination learning can be generalized to forms of hippocampal-dependent and hippocampalindependent representation that could support spatial learning. Thus, a "place" might be represented in terms of positional relations among environmental cues and the observer; if so, the representation of spatial relations should support flexible performance, for example, a capacity for navigation by novel routes. In accordance with our views on discrimination learning, this form of representation is dependent on hippocampal processing. Alternatively, a "place" might be represented in terms of the compound of environmental stimuli surrounding a location, like a "snapshot" of part of the environment; if so, even in the absence of relational processing, it should be possible for animals with hippocampal system damage to demonstrate place learning in repetitions of the acquired approach response. Such a pattern of results would indicate that learning for "places," as a category of stimulus materials, has no special status with regard to hippocampal processing and that, as with other types of learning, place learning requires hippocampal processing only when it puts a high demand on relational representation and flexible performance.

The present study focuses on memory representation in the open-field water maze, a place-learning task for which performance is highly dependent on distal visual cues (Morris, 1981). In the standard version of this task, the maze is composed of a circular swimming pool filled with an opaque solution of water and containing an escape platform submerged just under the water surface at a constant locus relative to multiple extramaze visual cues. On each training trial, the rat is placed at one of various start locations near the circumference of the maze and allowed to swim until it locates the escape platform. Over suc- 
cessive trials, normal rats learn to locate the platform more rapidly; eventually, they navigate directly to the platform from any start location (Morris, 1981, 1984).

Hippocampal system damage results in a severe and lasting impairment in acquisition of this task (Morris et al., 1982; Sutherland et al., 1983; Schenk and Morris, 1985). However, rats with hippocampal system damage might be able to learn the locus of the escape platform and escape as rapidly as normal rats, using the identical spatial cues, were they to be trained under conditions emphasizing an individual association between a place defined in terms of a set of extramaze stimuli and a single stereotyped swim trajectory. Furthermore, consistent with our observations on nonspatial discrimination learning, we believe that the memory representation supporting such learned performance in rats with hippocampal system damage would differ from that of intact rats. Specifically, whereas normal rats should be able to exploit their representation in novel situations, rats with hippocampal system damage should be impaired in tests other than repetition of the original learning event.

To test this prediction, we trained rats with fimbria-fornix lesions on the open-field water maze using procedures that encouraged rats to associate the extramaze cues with a particular swim trajectory and with consequent escape reinforcement. On each trial rats began from the same start location. In addition, the escape platform was visible on early trials, increasing the likelihood of swimming along the direct escape trajectory and decreasing their exposure to other parts of the pool. In later sessions, the visibility of the platform was "faded out" until, in the final pretest phase, rats were required to locatc a hidden platform using only extramaze cues. We predicted that, under these training conditions, rats with fornix lesions would be able to learn to swim directly to the platform and that ultimately their performance would be ostensibly indistinguishable from that of normal rats.

Then, to unmask the presumed differences in spatial representation, we conducted different types of "probe" trials presented within a series of standard training trials. Probe trials involved the use of novel start loci, elimination of the escape platform, deletion of some of the extramaze cues, and selective cue rotation. We predicted that normal rats would be able to use their representation of positional relations of environmental cues to locate the escape platform rapidly from novel start loci, but that rats with fornix lesions would be unable to use the representation gained during the training experience to navigate from a different view of the environment. It has been previously observed that intact rats can navigate successfully from novel start locations (Morris, 1981), indicating that their representation is flexible but, to our knowledge, no such test has been applied to rats with hippocampal system damage.

We also expected that the representation of rats with fornix lesions would be dependent to a greater degree than that of normal rats on those cues prominent from the view along the trained escape route. Thus, we predicted that the performance of rats with fornix lesions would be particularly affected when those cues were selectively altered. Conversely, in the probe test where the escape platform was removed but extramaze cues were unaltered, we expected that rats with fornix lesions would normally focus their search for the platform in the appropriate region of the pool.

In a final test, the ability of rats to acquire the conventional version of the water-maze task was assessed by moving the hidden escape platform to a new place in the pool and using multiple starting points. This version strongly encourages a representation of positional relations among environmental cues over development of individual representations for each starting point, since the latter would necessarily involve representations based on overlapping and conflicting cues and swim trajectories. We predicted that normal rats would readily acquire this version of the task, but that rats with fornix lesions would be severely impaired, and remain so over successive trials.

\section{Materials and Methods}

Subjects. Seventeen male Lister-hooded rats $(250-350 \mathrm{gm})$ were obtained from the breeding colony at the University of Edinburgh. They were individually houscd and kcpt on a 12-hr: 12-hr light-dark schedulc.

Surgery. Subjects were anesthetized with tribromoethanol (Avertin, $1 \mathrm{ml} / 100 \mathrm{gm}$, i.p.). The head was mounted in a Kopf stereotaxic instrument with bregma and lambda at the same height. In the group with intended fornix transections (FX rats; $n=7$ ), bilateral radiofrequency current was passed to maintain a temperature of $70^{\circ} \mathrm{C}$ for 1 min bilaterally with the tip of the electrode placed at each of several coordinates sites relative to bregma: A-0.3, L0.7, V4.2, and 4.0 and A-0.8, L1.7 $\mathrm{V} 4.4$, and 4.0 , and a midline lesion was made at coordinates A-0.3, L0.7 (at an angle of $10^{\circ}$ towards medial), V4.4, and 4.2. Animals were allowed to recover for $7-10 \mathrm{~d}$ before beginning training. Sham-operated subjects (SH rats; $n=10$ ) underwent the same anesthetic and surgical procedures, except that the electrode was not lowered fully into the fornix and no current was passed.

Histological verification of fornix lesions. After training, FX rats were deeply anesthetized, perfused with formal calcium, and the brain was removed. Frozen sections were taken at $30 \mu \mathrm{m}$ through the area of the lesion and stained with Cresyl violet, and lesions were evaluated for the amount of damage to the fornix and surrounding structures. In addition, the completeness of the fornix transection was evaluated in terms of its effects on AChE staining in the hippocampal formation itself (Namba et al., 1967). Our examination showed that the fornix was at least $90 \%$ destroyed in each case, and that the cholinergic input to the hippocampus was eliminated as revealed in the almost complete absence of $\mathrm{AChE}$ staining in all subdivisions of the hippocampal formation throughout its septo-temporal extent (Fig. 1). In some cases, the lesions extended into caudal portions of the medial and lateral septal nuclei. There were no substantial differences between rats as to the extent of fornix damage or AChE staining in the hippocampus.

Apparatus and training and testing procedures. Behavioral training and testing were conducted in Edinburgh using general procedures described in detail elsewhere (Morris, 1984), except as noted below. Briefly, the swimming pool was a $2-m$-diameter fiberglass cylinder painted white on the inside and filled to $28 \mathrm{~cm}$ from the top with $18^{\circ} \mathrm{C}$ water made opaque by the addition of fatted milk powder. The pool was situated in a $4.5 \mathrm{~m}^{2}$ room with various doors, posters, cabinets, and electrical fixtures visible from inside the pool (Fig. 2). Diffuse illumination was provided by indirect lighting from four 500-Watt floodlamp fixtures aimed towards the ceiling. Various escape platforms (see below) composed of Plexiglas cylinders with a flat metallic top were placed $32 \mathrm{~cm}$ from the edge of the pool in either the SW or NE quadrant. The rat could see the experimenter only as it was released to swim in the first few seconds of each trial.

Rats were trained and tested in 3 sequential experimental phases. First, they were trained to locate the escape platform after beginning each trial from the same start position. This phase will henceforth be called the constant-start condition. Second, mixed in with frequent trials identical to those of the constant-start condition, rats were given 1 of 3 types of probe tests: (1) novel-start tests that involved one of 6 start locations not previously used, (2) transfer tests where the escape platform was eliminated or moved, and (3) cue-alteration tests that involved elimination or rotation of some of the extramaze cues. In a third and final phase, rats were trained on a version of the task with the escape platform moved to a new location and with trials starting from any of 4 different positions. This phase was called the variable-start condition. Training and testing on each of these phases will be described in turn.

Training on the constant-start condition. In the early sessions of this phase of training, the escape platform was $10 \mathrm{~cm}$ in diameter and protruded $1 \mathrm{~cm}$ above the water surface in the SW quadrant. The walls of the cylinder were painted with 1-cm-wide vertical altemating black and white stripes. On each trial the rat was started facing the inner wall at 

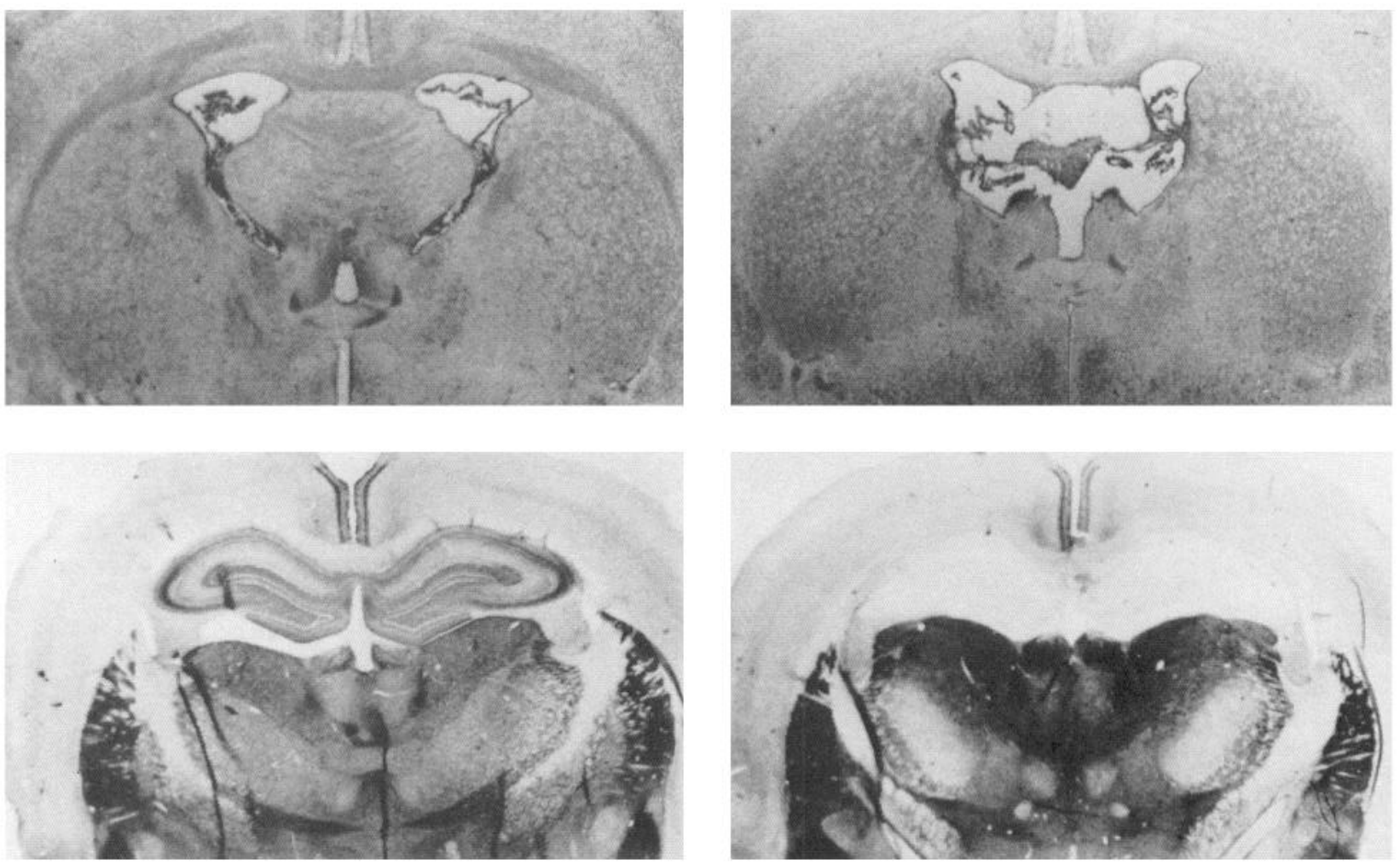

Figure 1. Photomicrographs of Nissl- (top) and AChE-stained (bottom) brain sections in SH (left) and FX (right) rats. Note elimination of the fornix in the Nissl section of the FX rat and consequent loss of AChE staining in the hippocampus.

the $S$ end of the pool. If the rat did not reach the escape platform within $120 \mathrm{sec}$, it was placed on the platform. Rats were allowed to remain on the platform for $30 \mathrm{sec}$ at the end of each escape, and successive trials were begun immediately thereafter. Rats were trained for 4 trials/d until they reached a criterion of escape latencies under $10 \mathrm{sec}$ on at least 3 trials on 2 successive days.

In succeeding sessions, training procedures were identical but the visibility of the escape platform was gradually faded out. At the beginning of this part of training the escape platform was a 20 -cm-diameter white disk protruding $0.5 \mathrm{~cm}$ above the water. Rats were required to reattain the performance criterion of 2 successive sessions each with escape latencies on at least 3 trials under $10 \mathrm{sec}$. In the following two 4-trial sessions, the escape platform was a cylinder, first of $15 \mathrm{~cm}$ diameter, then $10 \mathrm{~cm}$ diameter, protruding $0.5 \mathrm{~cm}$ above the water line. Finally, on this and all subsequent phases, the 10-cm-diameter escape platform was submerged $1 \mathrm{~cm}$ below the water surface. Rats were again trained to the performance criterion. The procedure used on these final trials of the constant start condition will be referred to as "regular" trials (see Fig. 2).

Probe tests. Three different types of probe tests were administered over a sequence of 6 daily testing sessions:

1. Novel-start tests. In each 6-trial session trials $1,2,4$, and 5 were regular trials. On trials 3 and 6 of each session, all procedures were identical to those of regular trials except that novel starting positions were used (Fig. $3 A$ ). Each of 6 novel start loci was used only once, in the following sequence: NE, NW, E, W, N, SE.

2. Transfer test. In a single session "transfer test" (Morris, 1984), the escape platform was removed and rats began the trials from the NW and were allowed to swim for $60 \mathrm{sec}$ before being taken from the pool. This test also involved a "novel" start, hence performance might in part have been dependent on a capacity for navigation. However, because both SH and FX rats usually located the platform in much less than $60 \mathrm{sec}$ (see below), the test primarily evaluated a rat's recognition of the place of escape rather than the accuracy of its initial trajectory.

On the following day, performance on regular trials was reinstated in a single session composed of 4 regular trials.

3. Cue-alteration tests. In a single six-trial session, trials $1,2,4$, and 5 were regular trials. On trial 3, 2 extramaze cues were removed (Fig.
$3 B$ ). These cues, a relay rack and a black curtain, were particularly prominent in the view from the $\mathbf{S}$ start locus looking in the direction of the escape platform. The start location on this trial was NE. On trial 6 , the same cues were rotated $180^{\circ}$ around the pool (Fig. $3 \mathrm{C}$ ). The start location was $\mathrm{N}$.

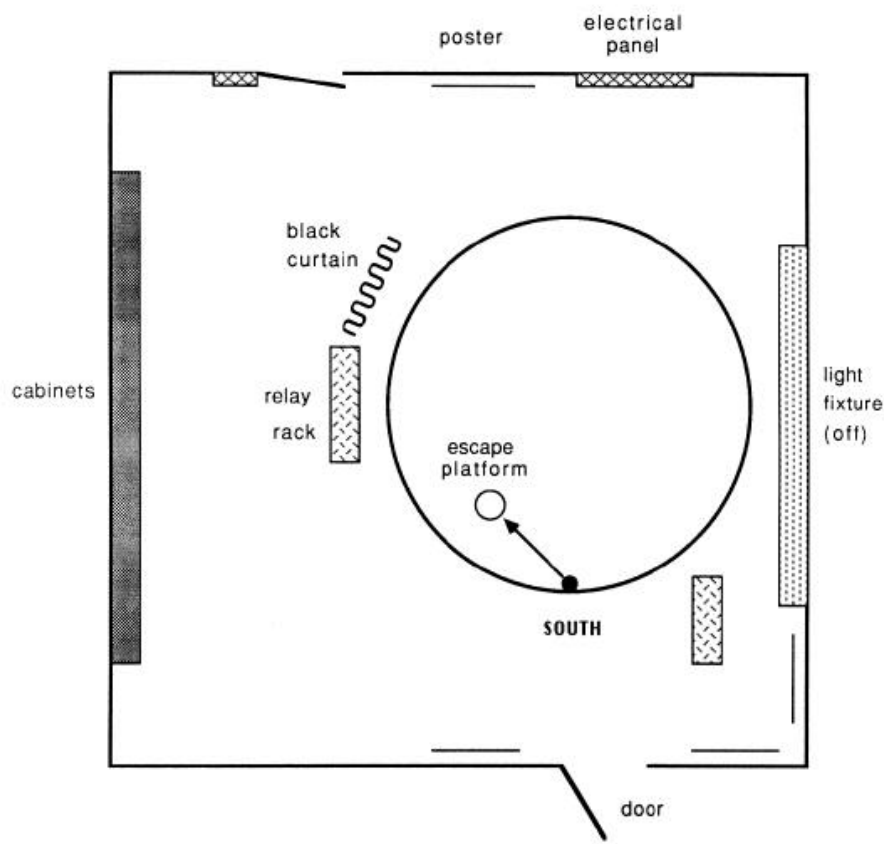

Figure 2. Schematic diagram of top view of cues in testing room that could be seen from inside open-field water maze. Arrow indicates the starting point and direction of minimal swim path in the constant-start condition. 
A. NOVEL-START

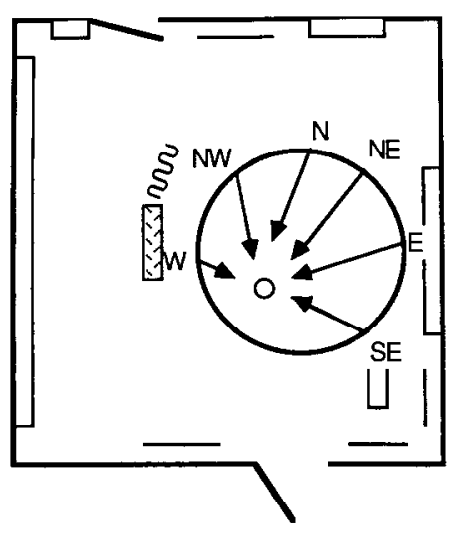

B. CUE-DELETION

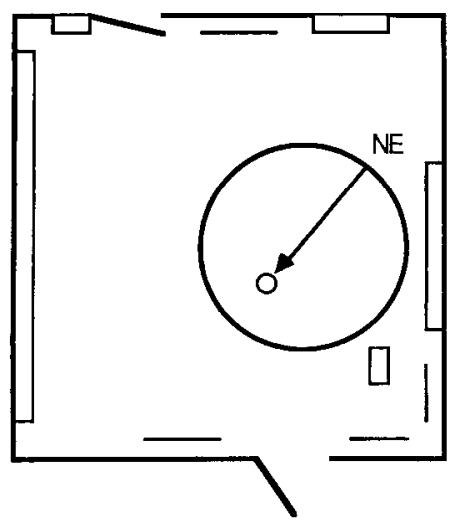

C. CUE-ROTATION

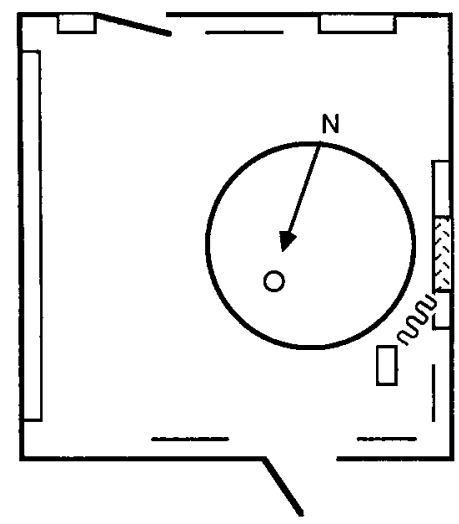

Figure 3. Schematic diagrams of the testing room indicating locations of cues, starting points, and minimal swim paths in different types of probe trials. For identification of cue symbols, see Figure 2.

Training on the variable-start condition. The location of the escape platform was moved to the NE quadrant and trials started from $S, N$, $E$, or $W$ according to a pseudo-random sequence. Three sessions were given on sequential days, each composed of 6 trials with a maximum escape latency of $60 \mathrm{sec}$.

Data analysis. Latencies of escape responses were timed with a stopwatch. In addition, a video-tracking system (Morris, 1984) was used to monitor the animal's position at $10 \mathrm{~Hz}$. For some trials, computer printouts of the escape path were used to estimate the heading error as the angular deviation from a direct path to the center of the escape platform measured at $35 \mathrm{~cm}$ (half the minimum possible distance on regular trials) from the starting point.

Performance on the transfer test was assessed by measuring the time spent in each of 4 angular quadrants of the pool centered on SW, NW, $\mathrm{NE}$, and SE. These measures were also taken on the first trial of the variable-start condition, when the platform was moved to a new location.

\section{Results}

Acquisition and performance on the constant-start condition Escape latencies for both SH and FX rats were high on the first trial, and all rats showed a gradual reduction in latencies over the first three sessions (Fig. $4 A$ ). IIowever, even under the visible-platform condition, FX rats were slower to reduce their escape latencies to that required for reaching the performance criterion. A 2-way analysis of variance (ANOVA) on escape latencies over the first 12 trials of this phase indicated that both groups reduced latencies over trials $(F=19.56, d f=11 / 165, p$ $<0.001)$, but SH rats had shorter escape latencies than FX rats $(F=7.97, d f=1 / 15, p<0.05)$, and $\mathrm{SH}$ rats reduced their escape latencies more rapidly than FX rats (i.e., there was a significant group by trials interaction; $F=2.85, d f=11 / 165, p<0.005$ ). A $t$ test comparing group performance on the number of sessions required to reach the performance criterion indicated that $\mathrm{SH}$ rats (mean $=3.0$ sessions) reached criterion more rapidly than FX rats (mean $=3.7$ sessions; $t=2.41, d f=15, p<0.025$ ).

The performance of FX rats on the first phase of platform fading was maintained in that the difference in cumulative number of sessions to criterion was the same $(\mathrm{SH}$ : mean $=5.0, \mathrm{FX}$ : mean $=5.7 ; t=2.41, d f=15, p<0.025$ ). But, as training proceeded, FX rats fell slightly farther behind, reaching the performance criterion on the final phase (platform submerged) in an average of 10.6 cumulative sessions compared to $\mathrm{SH}$ rats at an average of 9.4 sessions $(t=1.96, d f=15, p<0.05)$.

Even though FX rats reached the performance criterion in the constant-start condition, their average escape latencies were longer than those of SH rats, even at the end of each training phase. Thus, in the last session of visible-platform trials, $\mathrm{SH}$ rats had a mean latency of $2.2 \mathrm{sec}$ compared to $4.6 \mathrm{sec}$ for FX rats $(t=$ 1.93, $d f=15, p<0.05$ ). This was also observed during the criterion sessions when the platform was submerged ( $\mathrm{SH}$ : mean $=3.1, \mathrm{FX}:$ mean $=8.0, t=2.18, d f=15, p<0.025$ ), and throughout subsequent testing, although group differences were not statistically significant at every phase. For example, on the reinstatement trials given after the transfer tcst, $\mathrm{SH}$ rats had a mean escape latency of $2.2 \mathrm{sec}$ compared to $4.6 \mathrm{sec}$ for FX rats ( $t=1.56, d f=15$, NS). Moreover, these differences in mean scores are somewhat deceptive because the performance of FX rats was not uniformly impaired. An examination of the distribution of escape latencies among all postcriterion regular trials (Fig. $4 B$ ) indicated that $\mathrm{SH}$ and FX rats had comparable escape-latency distributions except at the longest latencies; FX rats had over 10 times the incidence of escape latencies over 10 sec.

Analyses of swim paths at several stages of training showed that nearly all long latency trials of FX rats could be characterized as "near misses," that is, trials on which they swam just to one side of the platform, requiring considerable additional time to return and escape. We examined these trials in 2 ways. First, the swim paths on each long latency trial were inspected visually. Second, an objective analysis of heading errors was used to quantify our subjective evaluations of near misses. If 
indeed the primary difference between $\mathrm{SH}$ and $\mathrm{FX}$ rats was the occurrence of near misses, errors in initial headings would be expected to be both small and very similar for SH and FX rats. This was precisely what was observed. As evaluated by the Watson-Williams test for circular data (Zar, 1974), mean absolute heading errors did not differ significantly between groups at the end of the visible-platform phase $\left(\mathrm{SH}\right.$ : mean $=6.5^{\circ}, \mathrm{FX}$ : mean $=4.3^{\circ}, F=1.01, d f=1 / 15$ ) or during the criterion trials on the submerged platform condition $\left(\mathrm{SH}\right.$ : mean $=5.6^{\circ}, \mathrm{FX}$ : mean $=11.3^{\circ}, F=1.43, d f=1 / 15$ ). Thus, on most trials the performance of $\mathrm{FX}$ rats was indistinguishable from that of $\mathrm{SH}$ rats and, even on those trials where FX rats had abnormally long escape latencies, they headed out in the correct direction.

\section{Performance on novel-start tests}

In contrast to their relatively normal asymptotic performance levels on regular trials, FX rats were markedly impaired in their ability to find the escape platform from novel-start locations. To provide a visual impression of the performance of both groups on these probe tests, the individual swim paths for trials that began from 2 opposite novel positions ( $W$ and $E$ ) are shown (Fig. 5). Most SH rats had little difficulty finding the escape platform from either start position. They headed out into the pool, began to move towards the escape locus almost immediately, and usually took a relatively direct route to the platform. In contrast, FX rats headed out into the pool in the wrong general direction as often as the correct one, and in many cases swam in a seemingly aimless path from either novel start location.

To permit direct comparisons of swim latencies across trials with considerably different minimal swim paths, the observed escape latencies for each start location were normalized by the ratio of the minimum swim distance for that start location to the minimum swim distance on a regular trial (Fig. 6). Performance scores for each rat were calculated as the mean normalized escape latency for the 6 novel-start trials and for the 6 regular trials just preceding each probe. A 2-way ANOVA on these scores indicated that escape latencies were longer on novel start trials than regular trials $(F=19.34, d f=1 / 15, p<0.001)$, that $\mathrm{FX}$ rats had longer escape latencies than $\mathrm{SH}$ rats $(F=30.19$, $d f=1 / 15, p<0.001$ ), and that the escape latencies of FX rats were disproportionately increased on novel-start trials (i.e., there was a significant groups by trial-type interaction; $F=11.37, d f$ $=1 / 15, p<0.005)$.

\section{Performance on transfer tests}

Performance of FX rats was indistinguishable from that of $\mathrm{SH}$ rats on the standard transfer test; both groups spent most of the time in the $\mathrm{W}$ half of the pool (Fig. $7 \mathrm{~A}$ ). To statistically compare the distribution of swim times among quadrants in $\mathrm{SH}$ versus FX rats, the observed time in each quadrant was transformed to the arcsine of the fraction of the total test period (Winer, 1971). A 2-way, repeated-measures ANOVA on these transforms indicated a significant difference among the proportion of time spent in different quadrants $(F-33.3, d f=3 / 45, p<$ 0.001 ), but the groups did not differentially distribute the proportion of swim time among the quadrants $(F=1.57$, $d f=$ $3 / 45)$.

The trial on which the platform was moved to the NE (the first trial of the variable-start condition) also served as a transfer test, since the rats were given no advance information on the new escape location and nearly all rats swam for the entire test period without finding the platform. The analysis of proportions
A. PHASE 1 ACQUISITION

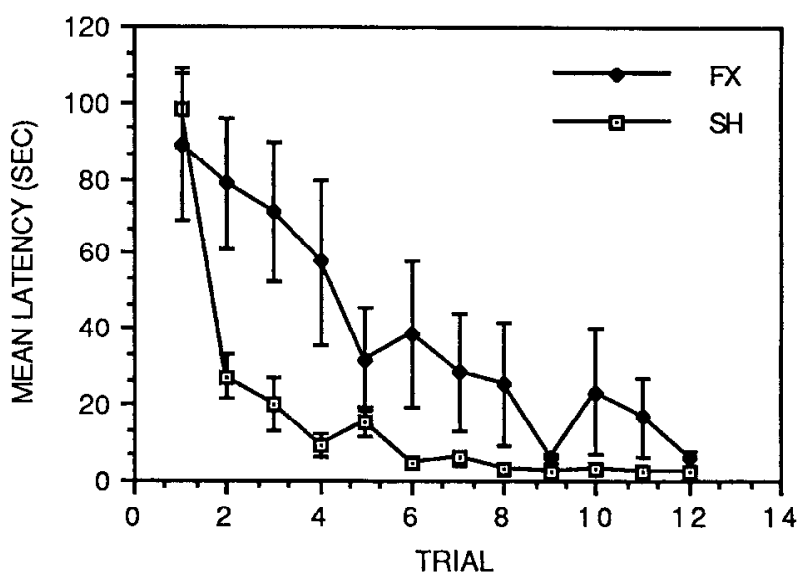

\section{B. POST-CRITERION LATENCIES}

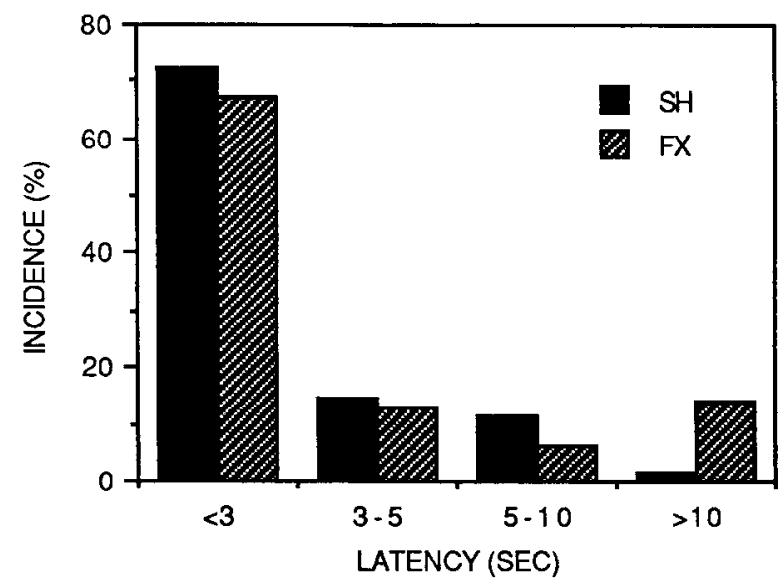

Figure 4. Acquisition in constant-start condition. A, Performance of groups $\pm \mathrm{SE}$ (vertical lines) in the initial (visible-platform) phase of training. $B$, Distribution of escape latencies during performancc of the learned approach response.

of swim time on this trial indicated that both groups confined their exploration even more closely to the original training locus that in the original transfer test, and that again the groups did not differ in search performance (Fig. $7 B$ ). As in the earlier transfer test, ANOVA on the transformed scores indicated a significant difference in swim times in different quadrants $(F=$ $38.15, d f=3 / 45, p<0.001$ ), but no significant difference between groups in the distribution of swim times among quadrants $(F=0.41, d f=3 / 45)$.

\section{Performance on cue-alteration tests}

Average escape latencies for FX rats were considerably elevated over those of $\mathrm{SH}$ rats both when those cues were deleted and when they were rotated $(F=17.64, d f=1 / 15, p<0.005)$, and the magnitude of the impairment was nearly identical for the 2 types of probe trials (Fig. 8A). Further insight into the nature of the impairment in FX rats on cue-rotation trials can be seen by comparison of their individual swim paths versus those of $\mathrm{SH}$ rats (Fig. 9). Most $\mathrm{SH}$ rats headed directly towards the center of the pool and swam in a relatively direct path to the platform. In contrast, most FX rats headed initially towards the rotated cues and had relatively circuitous routes to the platform. To 
A

NOVEL START - W

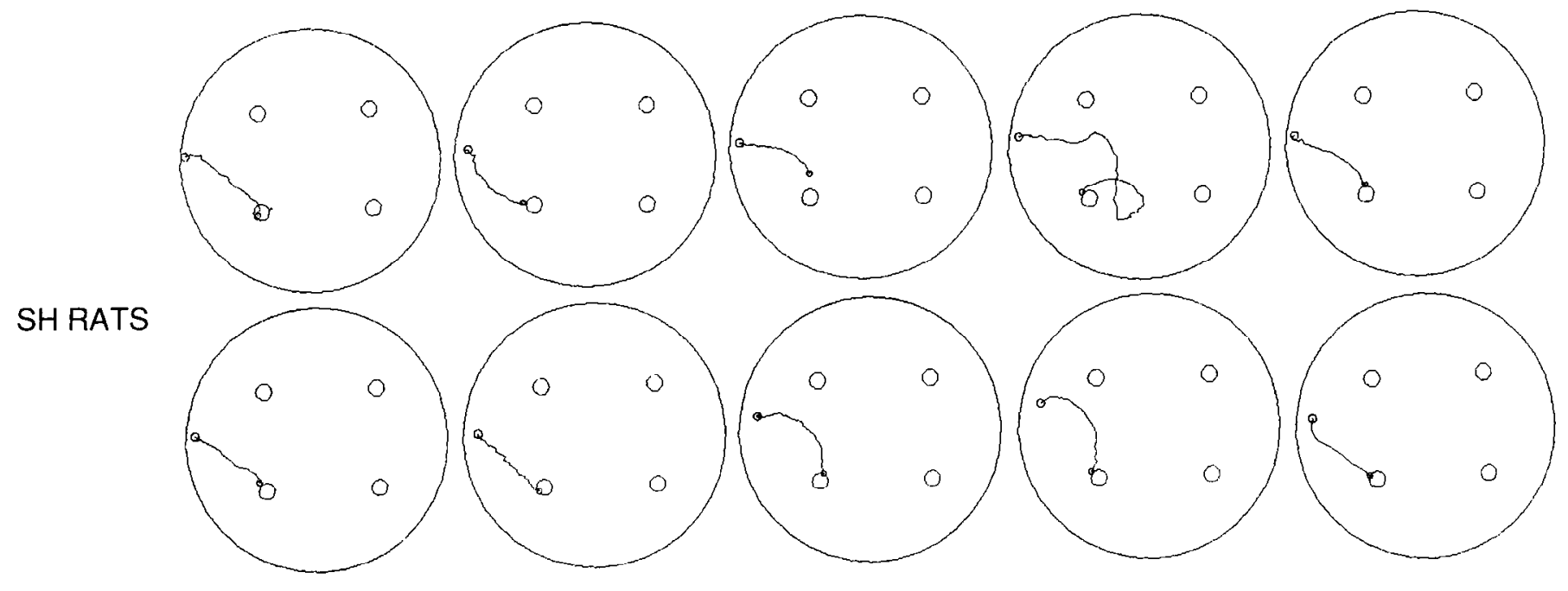

FX RATS
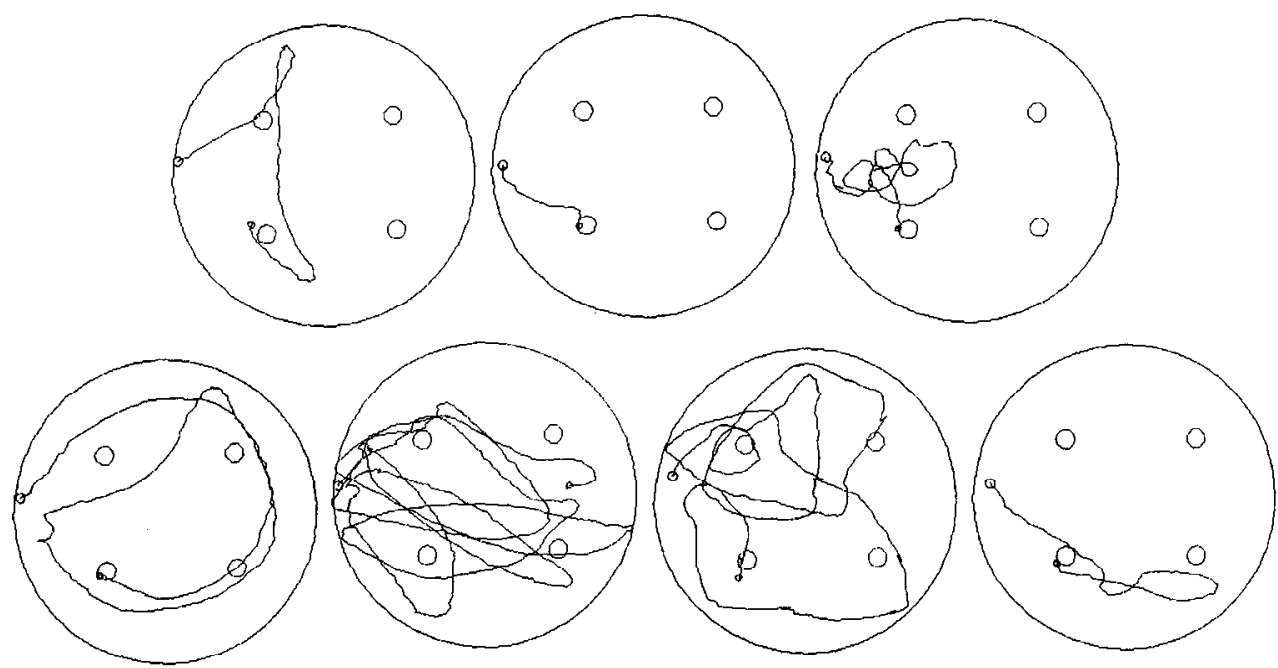

Figure 5. Tracings of individual swim paths on novel-start probe trials begun from the west $(W)$ and east $(E)$.

compare these behavior patterns more directly, we plotted the distribution of heading errors for both groups (Fig. $8 \mathrm{~B}$ ) and noted the error that would be predicted if rats were using only the rotated cues. (SW and $\mathrm{NE}$ are $50^{\circ}$ apart in heading viewed from the start location.) Headings in $\mathrm{SH}$ rats were generally near SW, the conventional location of the platform, although a few headings were slightly skewed towards the location of the rotated cues. In contrast, the headings of most of the FX rats were NE, in the direction of the rotated cues. The two FX rats with headings distant from NE did not find the escape platform in the 60 sec test.

\section{Acquisition in the variable-start condition}

When the escape platform was relocated and trials were started from different positions across trials, $\mathrm{SH}$ rats rapidly learned the new escape location but FX rats failed to decrease their escape latencies in the 3 sessions of testing (Fig. 10). A 2-way, repeated-measures ANOVA on average escape latencies in trial blocks composed of the first trial, the remainder of the first session (trials 2-6), the second session (trials 7-12), and the third session (trials 13-18), indicated that FX rats had significantly longer cscapc latencics $(F=22.53, d f=1 / 15, p<0.001)$ and that the differences in latencies became greater over trial blocks (i.e., there was a significant groups by trial-block interaction; $F=14.94, d f=3 / 45, p<0.005$ ).

\section{Discussion}

Our discussion of the pattern of performance in SH and FX rats addresses 2 issues with regard to hippocampal function in place learning: whether rats with damage to the hippocampal system are capable of "place" learning and what form of representation their place memory takes. 
B

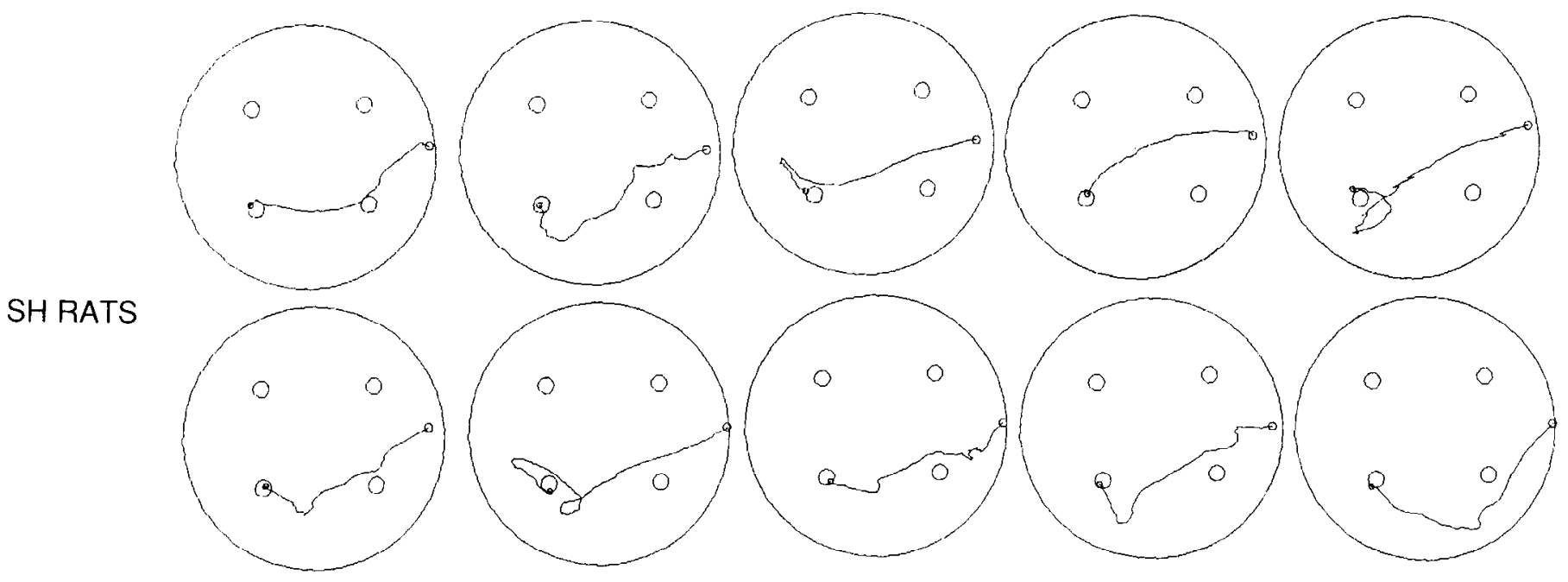

FX RATS

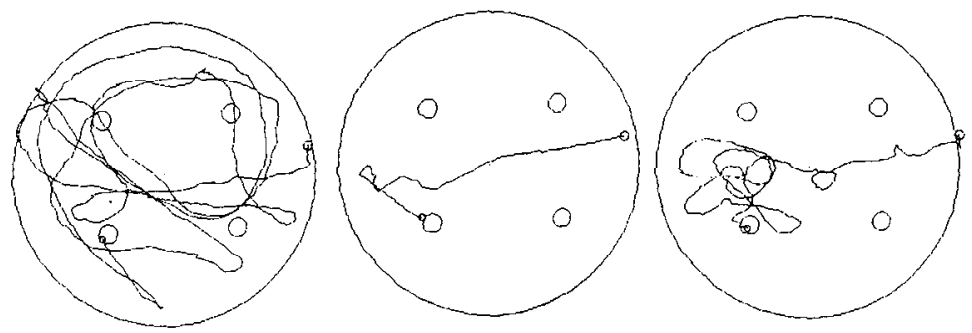

Figure 5. Continued.
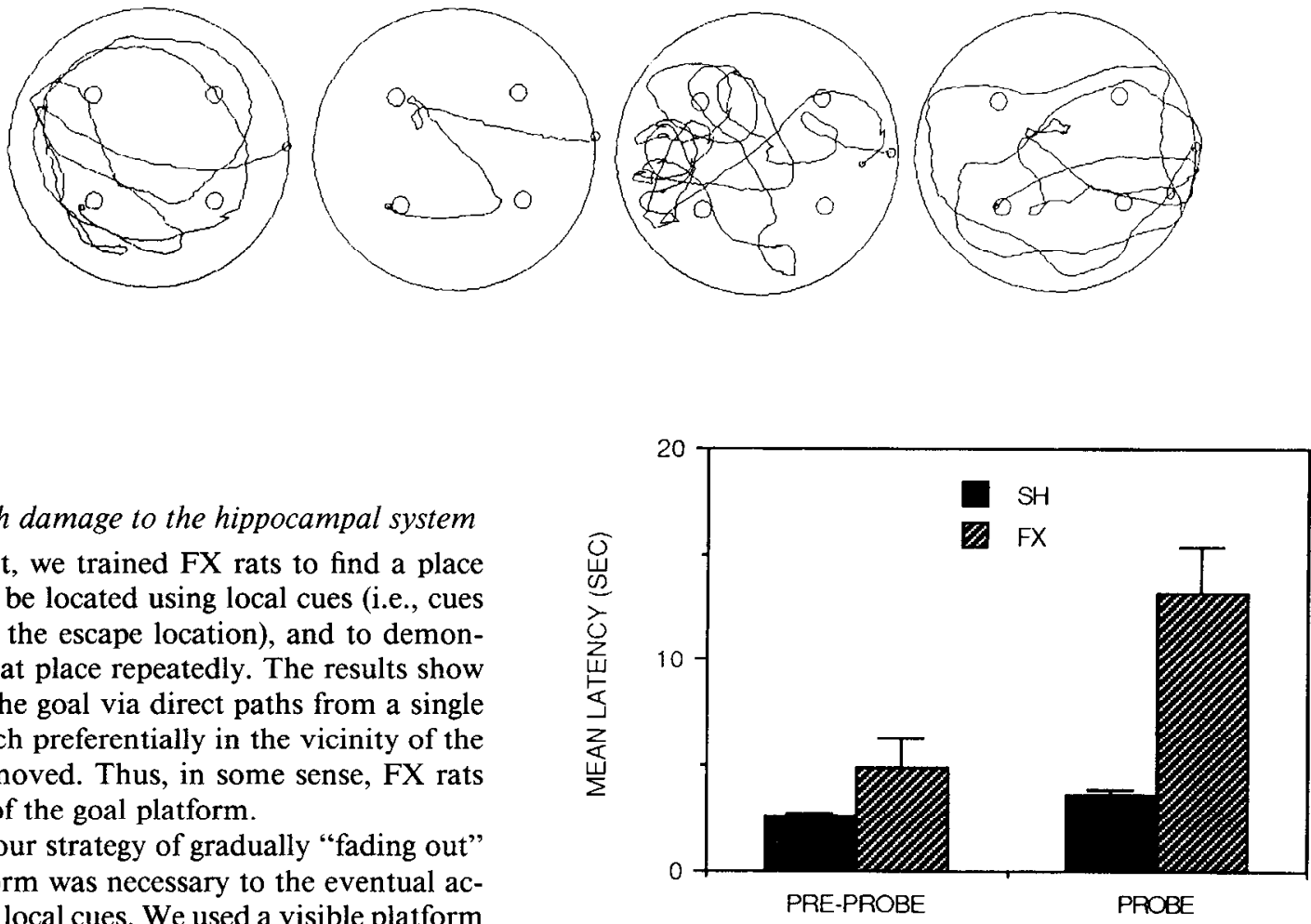

Place learning in rats with damage to the hippocampal system

In the present experiment, we trained FX rats to find a place that ultimately could not be located using local cues (i.e., cues spatially contiguous with the escape location), and to demonstrate their memory of that place repeatedly. The results show they learned to swim to the goal via direct paths from a single start location and to search preferentially in the vicinity of the platform when it was removed. Thus, in some sense, FX rats had learned the "place" of the goal platform.

It is not clear whether our strategy of gradually "fading out" the visibility of the platform was necessary to the eventual accurate navigation without local cues. We used a visible platform in the early training phases because rats have an initial tendency to swim towards an object that rises above the water surface, and thus using such a platform increased the likelihood of the

Figure 6. Performance in novel-start probe trials. Average escape latencies on regular trials presented just before each probe trial are compared with average escape latencies across the 6 novel starting positions. Vertical lines indicate SE. 


\section{A. TRANSFER TEST}

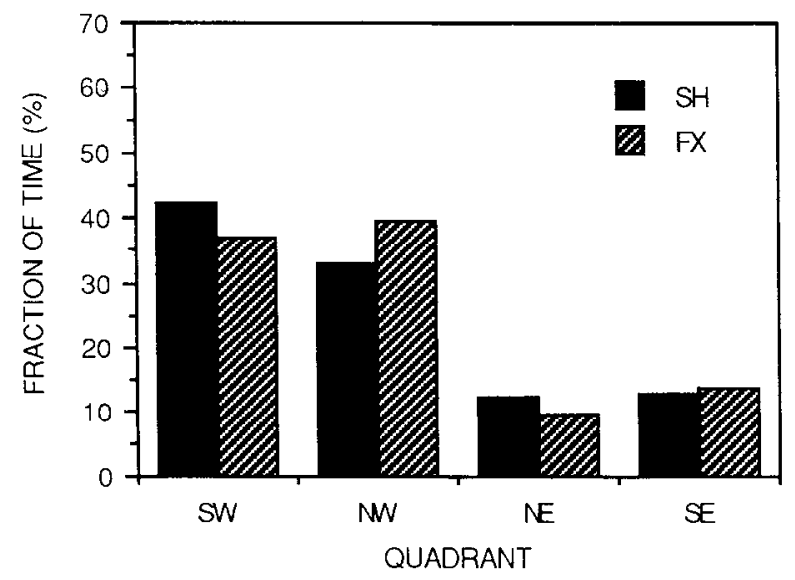

B. NEW PLACE - TRIAL 1

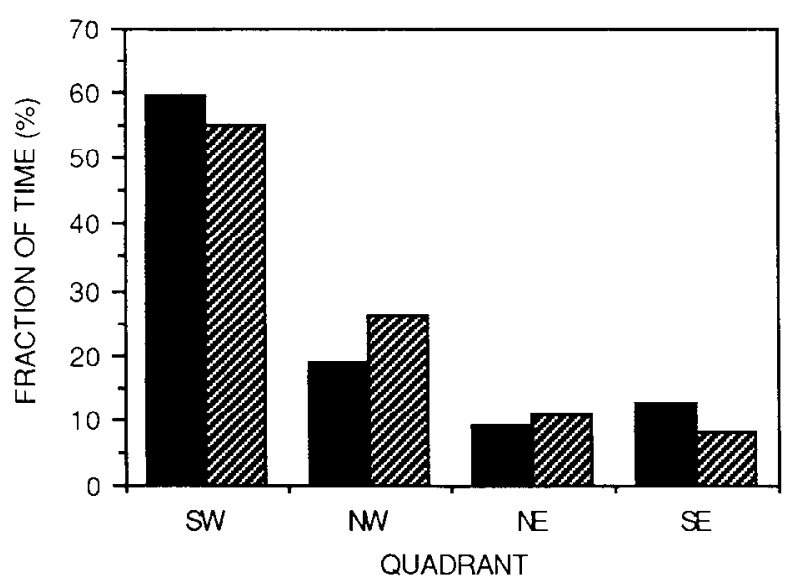

Figure 7. Distribution of swim times on transfer tests. $A$, Transfer test where no escape platform was present. $B$, Initial trial where the escape platform was moved to the NE.

direct swim trajectory being reinforced early in training. It is possible that $\mathrm{SH}$ and $\mathrm{FX}$ rats were developing different representations of the task even when the platform was visible, for as Morris (1981) showed, normal rats acquire a representation of the place of the escape platform, even when it is visible. The present experiment does not make clear whether FX rats also encoded distal cues at the outset of training, nor does it identify exactly when place learning occurred. However, regardless of when it happened, the eventual success of FX rats and their dependence on distal cues clearly indicates that they did ultimately learn some aspect of the "place" of escape.

The results of the probe tests indicate that the FX rats employed a memory representation based on distal cues rather than on a learned swim pattern. The swim paths taken during the transfer test did not replicate the specific turns or trajectories taken during acquisition. If FX rats simply learned to swim in a particular direction $\left(45^{\circ}\right.$ clockwise from the start), one would have expected that when started from the NW in the original transfer test, they would have focused their search for the platform in the NNE, but this was not observed. Rather, in both transfer tests, FX rats appeared to "search around" the previous locus of the platform to the same extent as SH rats. Furthermore,

\section{A. CUE-DELETION \& CUE-ROTATION TRIALS}

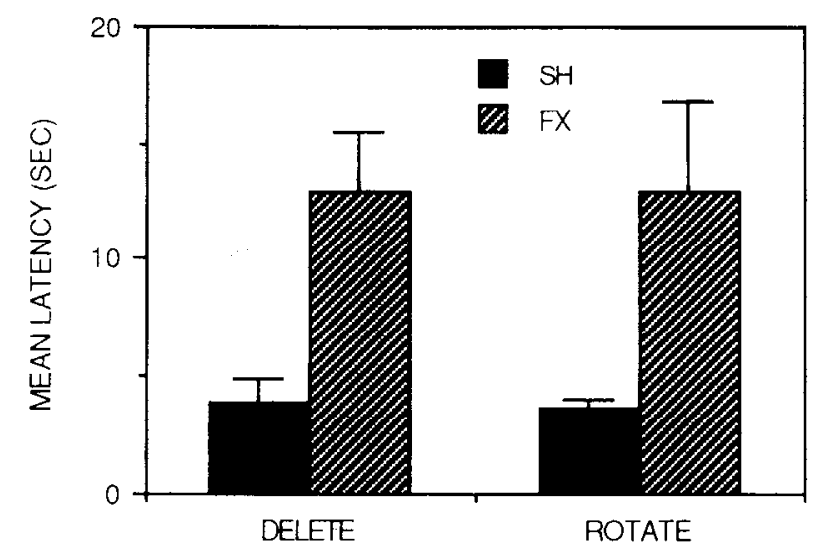

B. DIRECTION ON CUE-ROTATION TRIALS

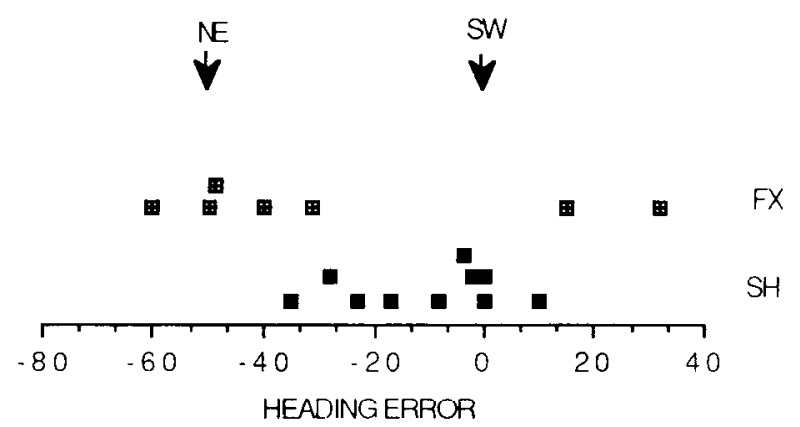

Figure 8. Performance on cue-alteration tests. $A$, Comparison of results on 2 types of cue-alteration tests. $B$, Distribution of individual heading errors in the cue-rotation test. Arrows indicate the score associated with a direct heading to the NE and SW platform positions. Vertical lines indicate SE. Heading errors are in degrees.

FX rats did not reliably begin novel-start tests by making a turn consistent with the swim trajectory of the training trials; their headings were unpredictable (see Fig. 4). Under the standard (variable-start) training conditions, rats with hippocampal system damage have been observed to use a "praxis" strategy; that is, they learn to circle the pool at roughly the radius of the platform as a successful albeit inefficient strategy for locating the platform (DiMattia and Kesner, 1988). No such swim strategy was evident under the present training conditions. Instead, FX rats acquired some knowledge about the location of the platform and used it successfully in the task for which they were trained.

In the constant-start condition, place learning in rats with hippocampal system damage can occur quite rapidly, although not as rapidly as in normal rats. The learning curve of FX rats had a more gradual slope than that of $\mathrm{SH}$ rats (see Fig. $3 A$ ), resulting in their requiring an average of just one more 4-trial session than $\mathrm{SH}$ rats to acquire the task. At criterion performance level, the performance of FX and SH rats was indistinguishable on the vast majority of trials. However, FX rats demonstrated occasional near misses, elevating their average escape latencies slightly during the regular trials which accompanied later test phases. These subtle differences in performance presage the larger distinctions in the nature of memory representation in FX and $\mathrm{SH}$ rats. 


\section{CUE ROTATION}

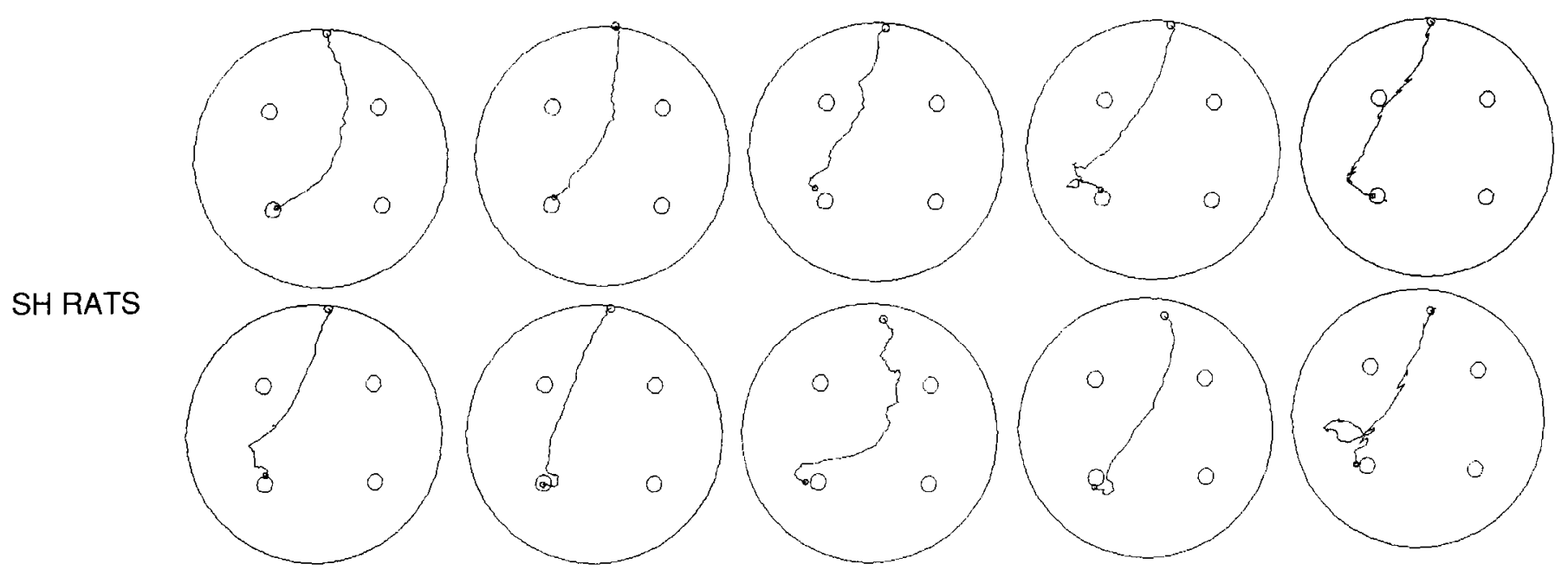

FX RATS
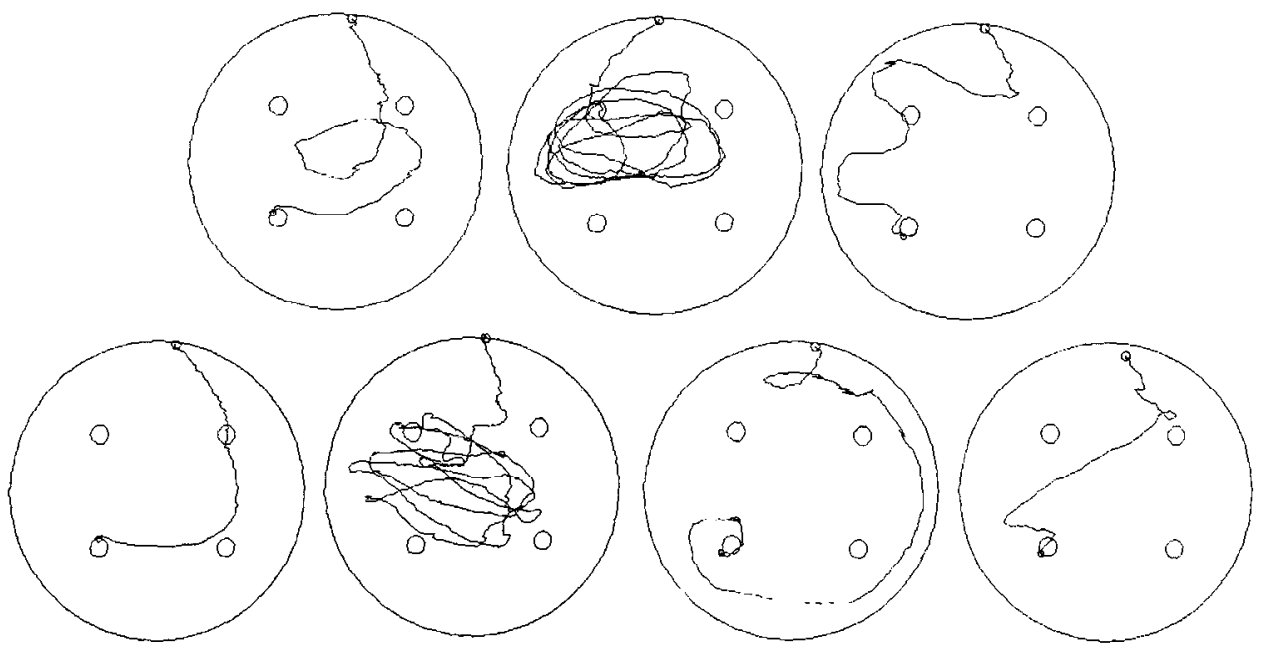

Figure 9. Tracings of individual swim paths on the cue-rotation probe trial.

Conditions for spared or impaired place learning and the nature of memory representation in rats with hippocampal system damage

In the variable-start condition, rats with hippocampal system damage demonstrate a severe and lasting impairment in place learning. In several other studies (e.g., Morris et al., 1982; Sutherland et al., 1983; DiMattia and Kesner, 1988; Schenk and Morris, 1985), and in the final test of the present study, rats with hippocampal system damage fail to learn the escape locus when a variable-start location is used. Others have attributed success or failure on maze learning to different types of cues available to guide performance (O'Keefe and Nadel, 1978). However, in the final stage of training in the present experiment, the available cues were identical and FX rats either performed the response as well as SH rats (the constant-start condition) or failed completely (the variable-start condition). Thus, we must conclude that FX rats used a different form of memory repre- sentation than $\mathrm{SH}$ rats, although both representations utilized the same sensory information.

How did their memory representations differ? In the constantstart condition, rats are reinforced consistently for a particular swim trajectory towards a particular complex of distal stimuli. While not preventing a relational representation, these conditions allow the formation of a simple association between an individual set of cucs and the behavior reinforced by successful escape. FX rats succeed in place learning under these conditions. In contrast, in the variable-start condition, rats are reinforced for multiple trajectories towards multiple-stimulus complexes. Successful navigation would seem impossible without taking into account the relations among the varying visual perspectives and swim trajectories. It is under these conditions that FX fail in place learning.

Even when rats with hippocampal system damage are successful in learning to navigate to a place, they use a different form of representation than normal rats, as revealed in their 


\section{NEW PLACE}

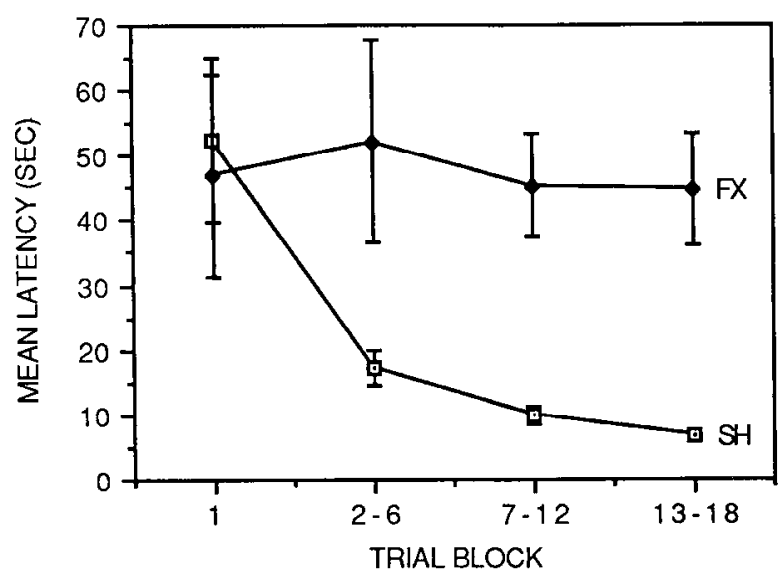

Figure 10. Acquisition in the variable-start condition. Scores are averages of daily trial blocks, except for the first trial, which was plotted separately to allow comparison of latencies in discovering a new platform location.

performance on probe trials. The results from each probe condition will be discussed in turn.

When a particular set of cues (a relay rack and curtain relatively near the escape site) and the start location were rotated $180^{\circ}, \mathrm{SH}$ rats continued to swim in a relatively direct trajectory to the escape platform. Thus their representation was not crucially dependent on a few cues nearest the escape site, nor was their performance disrupted by the unusual start locus. In contrast, most FX rats had strikingly different trajectories: they altered their swim trajectories consistent with the rotated cues and start locus. Also, FX rats required considerably longer to find the escape platform when the same cues were eliminated. Thus the representation of FX rats was abnormally dependent on the particular cues directly in front of the rats on regular trials (see Fig. 2). This abnormal dependency on cues near the site of reinforcement has been observed previously (Pico et al., 1985). But, in the present experiment, it does not appear that FX rats had only learned to approach those particular cues. Some FX rats headed neither to the actual escape site nor in the direction that would have been predicted by the rotated cue and start rotations. One explanation of their behavior is that they might simply have been "lost," that is, they did not recognize the environment after cue rotation. Furthermore, only one of the FX rats that headed towards the rotated-cues pursued that course the full distance predicted by the rotations (see Fig. 7; only $1 \mathrm{FX}$ rat crossed the NE platform site). Thus, for nearly all the FX rats, the way in which cues were represented differed from that of SH rats, but their representation included multiple environmental cues.

On probe trials using novel-start locations, SH rats consistently reoriented their swim trajectory towards the escape site. Thus, SH rats could use their memory representation "flcxibly" when challenged in a novel test situation. In contrast, FX rats required considerably longer to find the escape platform when required to begin from novel start locations. They had difficulty from a variety of novel starting points, including those from opposite directions where the most salient distal cues were near or far from the start locus (compare Figs. 4A, B). However, within the same series of trials, FX rats could reliably reproduce near-perfect swim trajectories when the conditions were iden- tical to those used during training. Thus, FX rats could not use their memory representation flexibly; they could reveal learning only in a repetition of the original act of acquisition.

\section{Implications for theories of hippocampal system involvement in learning and memory}

Early experiments on animals spawned by the characterization of human amnesia as "global" indicated a much more limited domain of impairment in nonhuman species. Some investigators suggested that the impairment in animals might be restricted to a particular cue (O'Keefe and Nadel, 1978) or response (Kimble, 1968) modality or to an attentional deficit (Douglas, 1967). However, subsequent studies have shown either impairment or sparing of learning capacities after hippocampal system damage, depending on the memory processing demands across stimulus and response modalities (Gaffan, 1974; Hirsh, 1974; Gray, 1979; Olton et al., 1979; Kesner, 1980; Mishkin et al., 1983; Rawlins, 1985; Sutherland and Rudy, 1989). Several theories have been generated to explain these findings. Each suggests that hippocampal processing is required for one form of memory representation and not another, but only some of these theories address hippocampal mechanisms for the flexible use of memories in novel situations. For example, Olton and colleagues (1979; for an additional similar account, see Kesner, 1980) suggested that the hippocampal system is required for working memory, which involves the processing of temporal relations among current stimuli and representation of recent experience. The working-memory account explains observed deficits in recognition memory after hippocampal system damage. However, it does not contain a mechanism for the generation of new behavioral responses based on recognition of familiar elements in novel situations, as required for navigation by a novel route from an unfamiliar start locus.

Other investigators have suggested that the hippocampal system is critical to learning that requires the disentanglement of cues with ambiguous associations in different contexts, such as in conditional learning paradigms (Hirsh, 1974; Winocur and Olds, 1978; Sutherland and Rudy, 1989). Based on one such formulation, Sutherland and Rudy suggested that successful place learning requires that various cue-response relations be disambiguated; they argued that the hippocampal system does this by creating unique "configural" cues composed of stimuli seen from separate views of the environment. Thus while individual cues have ambiguous associations with the correct response (e.g., walk to the left of $A$ when going from one start locus but to the right of A when going from another start locus), each configural cue has only one associated trajectory. This view can adequately account for the role of the hippocampus in learning the standard (variable start) version of the water maze, but appears to include no mechanism for understanding how the hippocampal system could successfully guide performance from a novel view of the environment.

In contrast to these accounts, the view proposed by O'Keefe and Nadel (1978) provides both a particular form of hippocampal-dependent representation that guides place learning and a mechanism for navigation from novel-start loci. In their view, the hippocampus establishes a cognitive map of the environment, a representation of spatial relations among environmental stimuli and the observer that supports spatial calculations necessary for successful navigation. Thus, while not explicitly addressed in its formulation, the cognitive-map account clearly incorporates a mechanism for navigation by novel routes. How- 
ever, in tasks such as that used in the present experiment, the cognitive-mapping account predicts either success or failure by FX rats according to the availability of local taxons versus distal spatial cues, respectively; the theory does not predict differential success or failure by FX rats in versions of this task guided by the same distal spatial cues. Furthermore, the cognitive-mapping account does not predict differential success or failure by FX rats across versions of other tasks guided by readily available nonspatial cues. Yet, as seen in the present experiment and those described below, both types of dissociations are sometimes observed.

\section{Tuwards a view of hippocampal function that incorporates the} data across behavioral paradigms and across species

Evidence exists that the hippocampal system is involved in memory processing for nonspatial, as well as spatial, relations among cues. For example, in a series of recent studies, Eichenbaum et al. (1988, 1989) examined impaired and spared learning capacities after hippocampal system damage within one particular paradigm: odor-discrimination learning in rats. They manipulated the memory processing demands so as to encourage or hinder certain types of processing on the identical stimulus materials. Rats with hippocampal system damage were severely impaired when odor cues were presented simultaneously, encouraging comparisons between cue and response alternatives. In contrast, when the discriminative stimuli were presented separately and the response requirement was to complete or discontinue a single behavioral act, thus hindering a representation based on relations between cues, rats with hippocampal system damage learned as rapidly or more rapidly than intact rats. To test directly the hypothesis that the memory representation of rats with hippocampal system damage was less flexible than that of intact rats, they challenged animals to use their acquired knowledge about familiar odors in novel discrimination problems composed of stimuli taken from previous problems and presented "mispaired" on probe trials (Eichenbaum et al., 1989). Normal rats sampled each odor in a discrimination problem and readily performed discriminations of familiar odor cues in novel pairings. In contrast, rats with hippocampal system damage sampled the multiple odors presented on each trial as a compound stimulus and their representation was inflexible in that it did not permit reference to the separate cue elements presented in novel pairings. Similarly, monkeys with hippocampal system damage initially trained to discriminate object pairs were later impaired in recognizing the pairings when the same objects were presented separately (Saunders and Weiskrantz, 1989).

Comparisons of learning capacities in animals with hippocampal system damage across behavioral paradigms is difficult, in great part due to the large number of differences between sensory, motivational, motor, and cognitive demands among commonly employed memory tasks. The open-field water maze would seem to have little in common with the above-described odor-discrimination tasks on any of these dimensions. Yet, there are striking general commonalities in the behavioral effects of hippocampal system damage on performance across these tasks. First, in both paradigms, one may observe either severely impaired or intact learning capacity depending on the representational demands employed, even when the identical stimulus materials are involved. Second, in both paradigms, learning is impaired when the task requirements encourage comparisons among multiple cues and a memory representation based on significant relations between them; learning capacity is spared when the task requirements hinder or eliminate the need for comparing cues and, instead, encourage approach responses to individual cues. Third, in both paradigms, even when the learning of specific items (places or odors) is impaired in animals with hippocampal system damage, there is a preserved capacity for acquisition of task procedures such as efficient swimming in the water maze and nose poking for rewards in the odor-discrimination task. Fourth, in both paradigms, even when learning is successful, animals with hippocampal system damage are unable to use their memories flexibly; they can demonstrate the acquired behavior only in repetition of the learning event.

These 4 properties of learning after hippocampal system damage can be extended to other learning and memory paradigms in rats, monkeys, and humans. Thus, several investigators have shown that rats with hippocampal system damage may demonstrate either severe impairment or intact learning for the identical materials depending on the task demands manipulated through the arrangement of cues in visually-guided maze learning (O'Keefe and Conway, 1980), the frequency of reward in radial-arm maze learning (Olton and Papas, 1979; Jarrard et al., 1984), the context of prior training in pattern-discrimination learning (Winocur and Olds, 1978), the assignment of cues in conditional sensory discrimination (Loechner and Weitz, 1987), and the reinforcement contingency in delayed-matching tasks (Rawlins et al., 1988). Monkeys with hippocampal system damage also demonstrate either impaired or intact learning with identical visual materials under varying representational demands (Gaffan, 1974; Gaffan et al., 1984; Zola-Morgan and Squire, 1985). In each of these tasks, the pattern of impaired and intact memory can be related to the requirement to compare information presented either simultaneously, such as in place or sensory discrimination, or sequentially, such as in working memory and delayed nonmatching tasks. Even when impaired in learning specific items, animals with hippocampal damage succeed in acquiring the procedural aspects of all these tasks. Finally, some of the most successful tasks for demonstrating memory impairment have an implicit demand for using information flexibly, for example, by making a response inconsistent with the behavior performed during acquisition of the material as in the working memory and delayed nonmatching tasks. Even in tasks where animals with hippocampal system damage are unimpaired in learning, they cannot use the acquired information flexibly in novel situations. For example, rats and monkeys even when unimpaired in acquiring sensory discriminations fail to recognize familiar discriminative cues in novel rcarrangcments (Eichenbaum et al., 1989; Saunders and Weiskrantz, 1989).

The common properties of impaired and intact learning capacity in animals also characterize the performance of human amnesics. For example, amnesic patients are impaired in a test that encourages active searching for representations and matching them to prompts, and doing so in a test unlike the learning experience. In contrast, they can recall the same words unconsciously when indirectly asked to simply repeat them (Graf et al., 1984). This pattern of performance in amnesics has been characterized as a distinction between an impaired representation in declarative memory, the memory system for facts and events that can be evoked by conscious recollection and can be used in a variety of ways, and a spared representation in procedural memory, a capacity for adaptations that can be demonstrated only in repetition of the learning event. The focus on 
relational processing and flexibility in accounts of amnesia in both humans and animals indicates that these properties are the hallmarks of memory dependent on the hippocampal system across species and across task modalities.

\section{References}

DiMattia BV, Kesner RP (1988) Spatial cognitive maps: differential role of parietal cortex and hippocampal formation. Behav Neurosci 102:471-480.

Douglas RJ (1967) The hippocampus and behavior. Psychological Bull $67: 416-442$.

Eichenbaum H, Fagan A, Cohen NJ (1986) Normal olfactory discrimination learning set and facilitation of reversal learning after combined and separate lesions of the fornix and amygdala in rats: implications for preserved learning in amnesia. J Neurosci 6:1876-1884.

Eichenbaum H, Fagan A, Mathews P, Cohen NJ (1988) Hippocampal system dysfunction and odor discrimination: impairment or facilitation depending on cognitive strategies. Behav Neurosci 102:331339.

Eichenbaum H, Mathews P, Cohen NJ (1989) Further studies of hippocampal representation during odor discrimination learning. Behav Neurosci 103:1207-1216.

Gaffan D (1974) Recognition impaircd and association intact in the memory of monkeys after transection of the fornix. J Comp Physiol Psychol 86:1100-1109.

Gaffan D, Gaffan EA, Harrison D (1984) Effects of fornix transection on spontaneous and trained non-matching by monkeys. Q J Exp Psychol 36B:285-303.

Graf P, Squire LR, Mandler G (1984) Amnesic patients perform normally on one kind of memory test for previously presented words. J Exp Psychol Learn Mem Cog 10:164-178.

Gray JA (1979) The neuropsychology of anxiety. Oxford: Oxford UP.

Hirsh R (1974) The hippocampus and contextural retrieval of information from memory: a theory. Behav Biol 12:421-444.

Jarrard L, Okaichi H, Goldschmidt R, Stewart O (1984) On the role of hippocampal connections in place and cue tasks: comparisons with damage to hippocampus. Bchav Neurosci 98:946-954.

Kesner RP (1980) An attribute analysis of memory: the role of the hippocampus. Physiol Psychol 8:189-197.

Kimble DP (1968) Hippocampus and internal inhibition. Psychological Bull 70:285-295.

Loechner KJ, Weisz DJ (1987) Hippocampectomy and feature-positive discrimination. Behav Brain Res 26:63-73.

Mishkin M, Malamut B, Bachevalier J (1983) Memories and habits: two neural systems. In: The neurobiology of learning and memory (McGaugh JL, Lynch G, Weinberger N, eds). New York: Guilford.

Morris RGM (1981) Spatial localization does not require the presence of local cues. Learn Motiv 12:239-260.

Morris RGM (1984) Developments of a water-maze procedure for studying spatial learning in the rat. J Neurosci Meth 11:47-60.
Morris RGM, Garrud P, Rawlins JNP, O'Keefe J (1982) Place navigation impaired in rats with hippocampal lesions. Nature 297:681683.

Namba T, Nakamura T, Grob D (1967) Staining for nerve fiber and cholinesterase activity in fresh fr sections. Am J Clin Path 47:74-77.

O'Keefe J, Conway DH (1980) On the trail of the hippocampal engram. Physiol Psychol 2:229-238.

O'Keefe J, Nadel N (1978) The hippocampus as a cognitive map. Oxford: Oxford UP.

Olton DS, Feustle WA (1981) Hippocampal function required for nonspatial working memory. Exp Brain Res 41:380-389.

Olton DS, Papas BC (1979) Spatial memory and hippocampal function. Neuropsychologia 17:669-682.

Olton DS, Becker JT, Handlemann E (1979) Hippocampus, space, and memory. Brain Behav Sci 2:313-365.

Pico RM, Gerbrandt LK, Pondel M, Ivy G (1985) During stepwise cue deletion, rat place behaviors correlate with place unit responses. Brain Res 330:369-372.

Rawlins JNP (1985) Associations across time: the hippocampus as a temporary memory store. Brain Behav Sci 8:479-496.

Rawlins JNP, Maxwell TJ, Sinden JD (1988) The effects of fornix section on win-stay/lose-shift and win-shift/lose-stay performance in the rat. Behav Brain Res 31:17-28.

Ross RT, Orr WB, Holland PC, Berger TW (1984) Hippocampectomy disrupts acquisition and retention of learned conditional responding. Behav Neurosci 2:211-225.

Saunders RC, Weiskrantz L (1989) The effects of fornix transection and combined fornix transection, mammillary body lesions and hippocampal ablations on object pair association memory in the rhesus monkey. Behav Brain Res 35:85-94.

Schenk F, Morris RGM (1985) Dissociation between components of spatial memory in rats after recovery from the effects of retrohippocampal lesions. Exp Brain Res 58:11-28.

Sutherland RJ, Rudy JW (1989) Configural association theory: the role of the hippocampal formation in learning, memory, and amnesia. Psychobiology 17:129-144.

Sutherland RJ, Wishaw IQ, Kolb B (1983) A behavioral analysis of spatial localization following electrolytic, kainate- or colchicine-induced damage to the hippocampal formation in the rat. Behav Brain Res 7:133-153.

Winer BJ (1971) Statistical principles of experimental design. New York: McGraw-Hill.

Winocur G, Olds J (1978) Effects of context manipulation on memory and reversal learning in rats with hippocampal lesions. J Comp Physiol Psychol 92:312-321.

Zar JH (1974) Biostatistical analysis. Englewood Cliffs, NJ: PrenticeHall.

Zola-Morgan S, Squire LR (1985) Medial temporal lesions in monkeys impair memory on a variety of tasks sensitive to human amnesia. Behav Neurosci 99:22-34. 